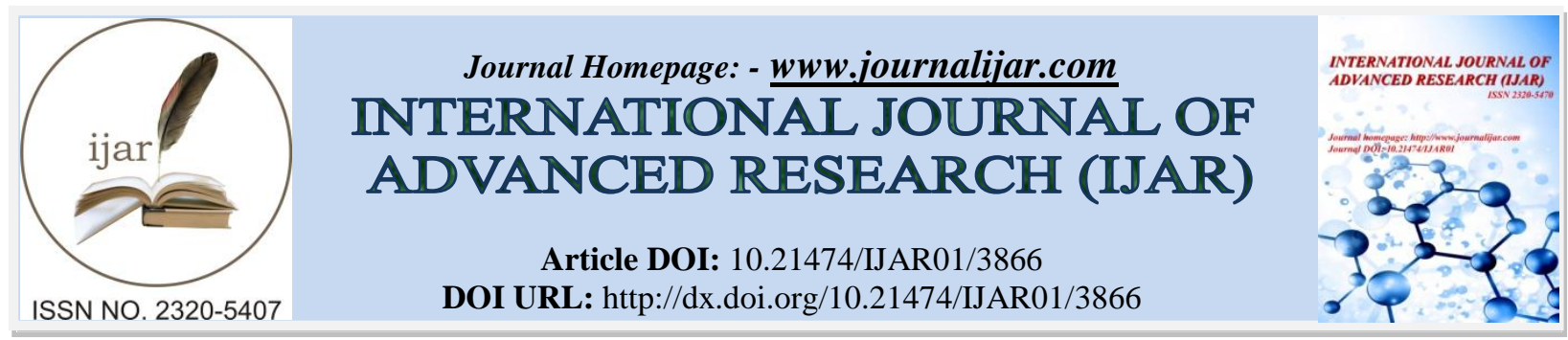

RESEARCH ARTICLE

\title{
GREEN SYNTHESIS OF SILVER NANOPARTICLES USING Citrus reticulata FRUIT PEEL AQUEOUS EXTRACT AND THEIR ANTIBACTERIAL ACTIVITY.
}

\author{
Shanmugavadivu. $\mathbf{M}^{1}$, Selvam Kuppusamy ${ }^{2}$ \\ 1. Department of Biotechnology, Dr. N.G.P. Arts and Science College, Coimbatore, Tamilnadu, 641048, India. \\ 2. Department of Botany, Periyar University, Periyar Palkalai Nagar, Salem, Tamil Nadu -636011, India'
}

\section{Manuscript Info}

Manuscript History

Received: 08 February 2017

Final Accepted: 05 March 2017

Published: April 2017

Key words:-

Citrus reticulata peels, silver nanoparticles, XRD, SEM, antibacterial activity

\begin{abstract}
Environmental friendly approach or green chemistry synthesis of metallic nanoparticles has become new growing branch in nanobiotehnology. In this present work a simple and environmental friendly silver nanoparticles (AgNPs) were prepared using Citrus reticulata fruit peels aqueous extract as the reducing agent guided by the principles of green chemistry. The fruit peels aqueous extract was challenged with silver nitrate solution for the production of AgNPs in room temperature. The crystalline phase and morphology of AgNPs were determined from UV-Vis spectroscopy, Fourier transform infrared (FTIR) spectra, X-ray diffraction (XRD), Scanning Electron Microscopy (SEM) and Energy Dispersive X-ray Spectroscopy (EDX). The UV-Vis spectrum indicated that the surface plasmon broad peak was observed at $466 \mathrm{~nm}$ after $6 \mathrm{hr}$ of incubation. XRD spectrum revealed that the average size of Citrus reticulata fruit peels aqueous extract mediated AgNPs obtained approximately $24 \mathrm{~nm}$ by using the Debye-Scherrer equation. SEM image showed uniformly distributed AgNPs on the surface of the cell with high agglomeration. EDX analysis revealed that the presence of silver which was confirmed by the $\mathrm{Ag}$ peak at $3.28 \mathrm{keV}$. In addition, the Citrus reticulata fruit peels aqueous extract mediated AgNPs loaded discs were tested for antibacterial properties against Streptococcus pyogenes, Staphylococcus aureus, Bacillus subtilis Escherichia coli, Salmonella paratyphi and Klebsiella pneumoniae and found that the obtained metallic AgNPs have a good antibacterial activity and can be used for biological applications.
\end{abstract}

Copy Right, IJAR, 2017,. All rights reserved.

\section{Introduction:-}

Nanoparticles are aromatic or molecular aggregates with at least one dimension between 1 and 100nm that can drastically modify their physic-chemical properties compared to the bulk material (Karnani and Chowdhary, 2013). They are the fundamental building blocks of nanotechnology. The most important and unique property of nanoparticles is that they exhibit larger surface to volume ratio. The most effectively studied nanoparticles today are those made from noble metals, in particular Ag, Pt, Au and Pd (Gurunathan et al., 2009; Jain et al., 2009).The synthesis of metal and semiconductor nanoparticles has attracted considerable attention from physicists, chemists, materials scientists and engineers owing to their potential applications in catalysis, biosensing, recording media and 
electronics (Sathyavathi et al., 2010). Among the above four noble metal, silver nanoparticles play a significant role in the field of biological systems, living organisms and medicine (Parashar et al., 2009).It is observed that silver nanoparticles do not affect living cells so they can be used as an effective anti-microbial agent as they get attached to the cell wall and disturb cell wall permeability and cellular respiration (Singh et al., 2008). Due to this property silver containing particles are used in textile fabrics, as food additives and in packaging to eliminate microorganisms (David et al., 2010). Therefore design and development of simple, one-step, non toxic and eco-friendly method for the production of multifunctional silver nanoparticles is of greatest interest to excel their applications.

Numerous methodologies are formulated in the past to synthesize silver nanoparticles of particular size and shape depending on their specific requirements. The usage of toxic chemicals and solvents in the synthesis of silver nanoparticles limits their application in the clinical fields. So the biopreparation of nanoparticles as an emerging field of the intersection of Nanotechnology and Biotechnology has received increased attention due to a growing need to develop environmentally benign technologies in silver nanoparticles synthesis (Madhumitha and Selvaraj, 2013).

The process for making silver nanoparticles using plant extracts is readily available and less expensive. The plant extracts act as reducing and stabilizing agents in the production of nanoparticles. This is because different plant extracts contain different concentrations of organic reducing agents (Mukunthan and Balaji, 2012). Though, plant mediated synthesis of silver nanoparticles are considered as safe, eco-friendly and cost effective, they also have some drawbacks in using the sustainable plant resources (Haverkamp and Marshall, 2009). For this reason, in the present study we used fruit peel extract of Citrus reticulata as alternate source.

The synthesized nanoparticles were confirmed by colour changes and characterized by UV-Visible spectroscopy. Fourier transform infrared (FTIR) spectral measurements were carried out to identify the potential biomolecules. The morphology of the nanoparticles was observed by SEM (Scanning Electron Microscope). The crystalline nature of Ag nanoparticles was confirmed by X-ray diffraction analysis. Furthers its efficacy to inhibit different pathogenic bacterial growth were evaluated against human pathogens by disc diffusion method.

\section{Materials and Methods:-}

\section{Preparation of Citrus reticulata fruit peels extract:-}

Citrus reticulata fruits were collected from the local market nearby our college. The fresh and healthy Citrus reticulata fruits peel were collected, rinsed thoroughly first with tap water for 5-10 min followed by distilled water for 10-20 min to remove all the dust and unwanted visible particles, fruit peels were cut into small pieces and dried at room temperature. To prepare the aqueous extract of Citrus reticulata fruits peel, $20 \mathrm{~g}$ of thoroughly washed and finely chopped fruit peels were added to $100 \mathrm{ml}$ of sterile distilled water in a $250 \mathrm{ml}$ Erlenmeyer flask and then boiled for 10min in heating mantle. The solution was then removed from the head source and left at room temperature. Following this step the extract was filtered through Whatman filter paper No.1 and used as such for silver nanoparticles synthesis.

\section{Synthesis of silver nanoparticles using Citrus reticulata fruit peel extract:-}

The aqueous solution of $1 \mathrm{mM}$ concentration silver nitrate $\left(\mathrm{AgNO}_{3}\right)$ was prepared to synthesize silver nanoparticles from Citrus reticulata fruit peel extract. $5 \mathrm{ml}$ of Citrus reticulata fruit peel aqueous extract was slowly added to $100 \mathrm{ml}$ of aqueous solution of $1 \mathrm{mM} \mathrm{AgNO}_{3}$ while stirring, for reduction into $\mathrm{Ag}$ ions. The colour change of the reaction mixture from faint yellow to yellowish brown to reddish brown to colloidal brown was monitored after $6 \mathrm{hr}$ of incubation at room temperature.

\section{Characterization of Silver Nanoparticles:-}

The bio reduction of $\mathrm{Ag}+$ ion in aqueous solution was monitored with the help of $\mathrm{UV}$-visible spectroscopic analysis. UV-Visible spectroscopy analysis of silver nanoparticles synthesized using Citrus reticulata fruit peels were carried out as a function of time needed for bioreduction at room temperature on UV-2600 series Shimadzu spectrophotometer between wavelengths of 300 to $800 \mathrm{~nm}$ at a resolution of $1 \mathrm{~nm}$. Fourier transform infrared (FTIR) spectra for green synthesised silver nanoparticles was recorded on a Shimadzu FTIR spectrometer 8000 series, with $\mathrm{KBr}$ in the wavenumber region of $4,000-400 \mathrm{~cm}^{-1}$. Crystalline nature of the nanoparticles was analyzed by XRD at $2 \theta$ ranges from 20 to $80^{\circ} \mathrm{C}$ using X'Pert Pro X-ray diffractometer (PAN analytical BV, The Netherlands) equipped with $\mathrm{Cu} / \mathrm{K} \alpha$ radiation source using $\mathrm{Ni}$ as filter at a setting of $30 \mathrm{kV} / 30 \mathrm{~mA}$. The morphology and elemental 
composition of the synthesised silver nanoparticles was identified by Scanning Electron Microscopy (SEM, JEOL JSM-6390) along with Energy Dispersive X-ray Spectroscopy (EDS, Model No. 9582, Oxford Instruments) operating at an accelerating voltage of $20 \mathrm{kV}$.

\section{Antibacterial Activity:-}

The biosynthesized silver nanoparticles using Citrus reticulata fruit peels aqueous extract were tested for antibacterial activity by disc diffusion methods against human pathogenic organisms such as Streptococcus pyogenes, Staphylococcus aureus, Bacillus subtilis Escherichia coli, Salmonella paratyphi and Klebsiella pneumoniae. Pure cultures of bacterial test organisms were grown in nutrient broth at $37^{\circ} \mathrm{C}$ for $24 \mathrm{~h}$. About $200 \mu \mathrm{L}$ of aliquot of each strain $(1 \times 106 \mathrm{cfu} / \mathrm{mL})$ was spread uniformly onto the individual pre-sterilized Mueller Hinton agar plates using sterile cotton swabs and allowed to dry for 10 to $15 \mathrm{~min}$. On other side, Whatman No. 1 filter paper discs ( $3 \mathrm{~mm}$ in diameter) were prepared and coated with $50 \mu \mathrm{l}$ of silver nanoparticles. The silver nanoparticles coated filter paper discs were placed on the surface of each cultured plate, Chloramphenicol disc was used as positive control and Citrus reticulata fruit peels aqueous extract was used as negative control. Then, the petridishes were incubated at $37^{\circ} \mathrm{C}$ for $24 \mathrm{~h}$. After incubation, the inhibition zones were measured in millimeters.

Figure 1:- Biosynthesis of AgNPs using Citrus reticulata fruit peels aqueous extract a) Citrus reticulata fruit peels aqueous extract b) $1 \mathrm{mM} \mathrm{AgNO}_{3}$ c) Reaction mixture after $6 \mathrm{hr}$ incubation

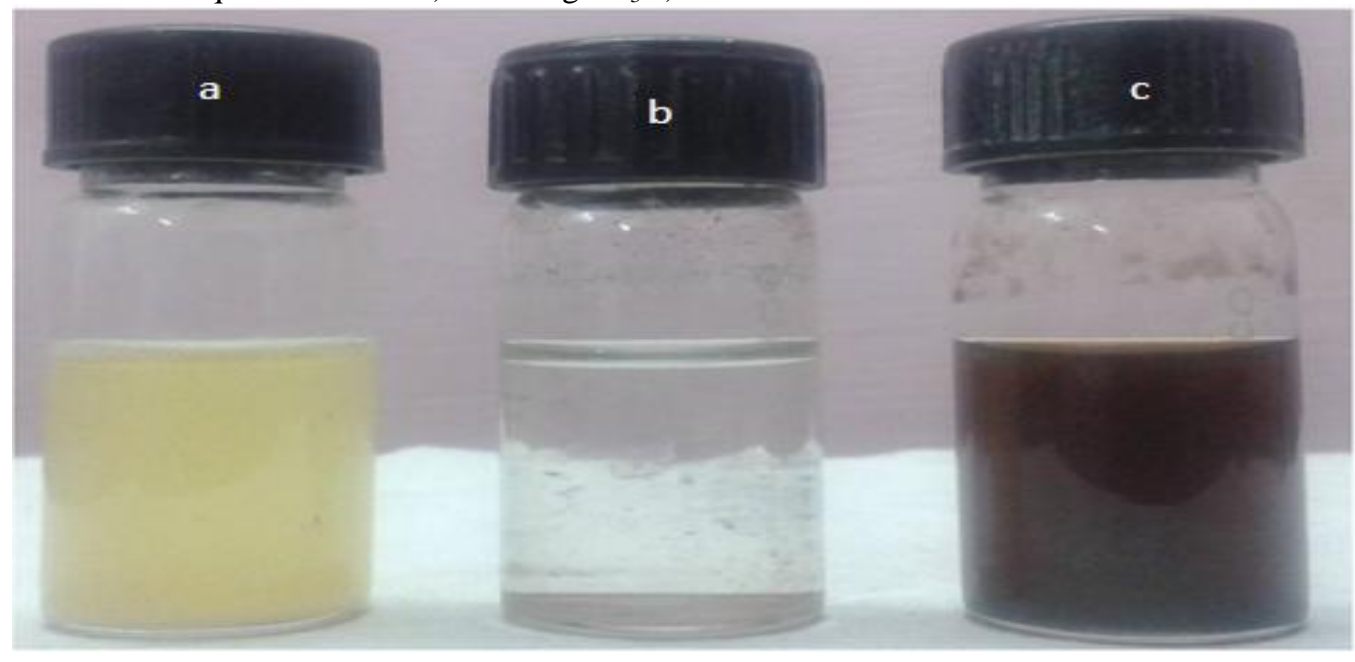

Figure 2:- UV Spectrum of biosynthesized Silver nanoparticles.

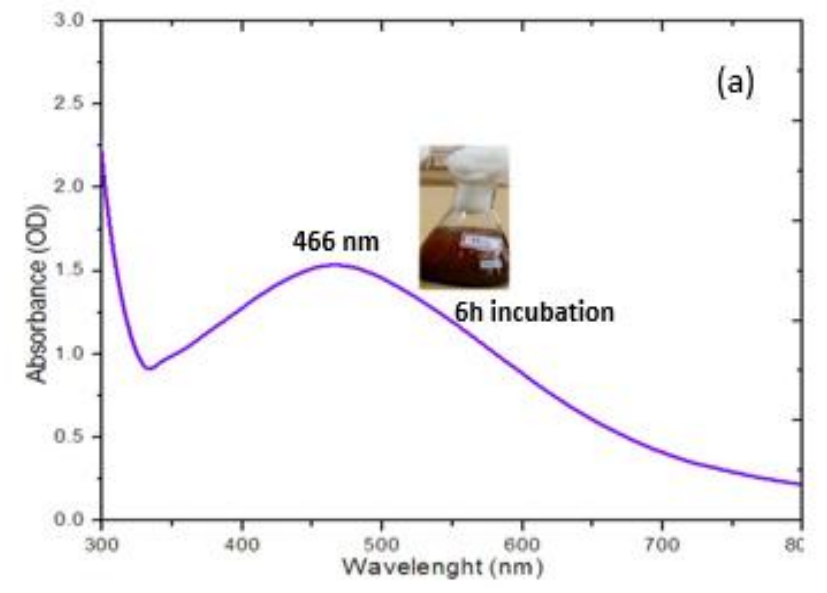


Figure 3:- FTIR Spectrum of Silver nanoparticles.

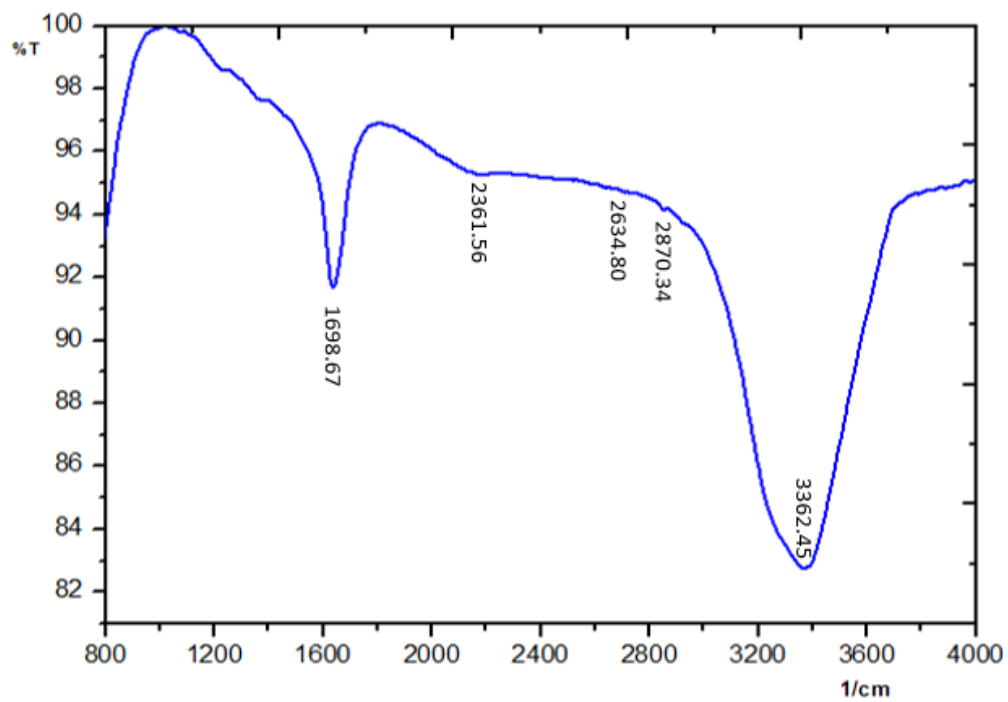

Figure 4:- XRD analysis of Silver nanoparticles

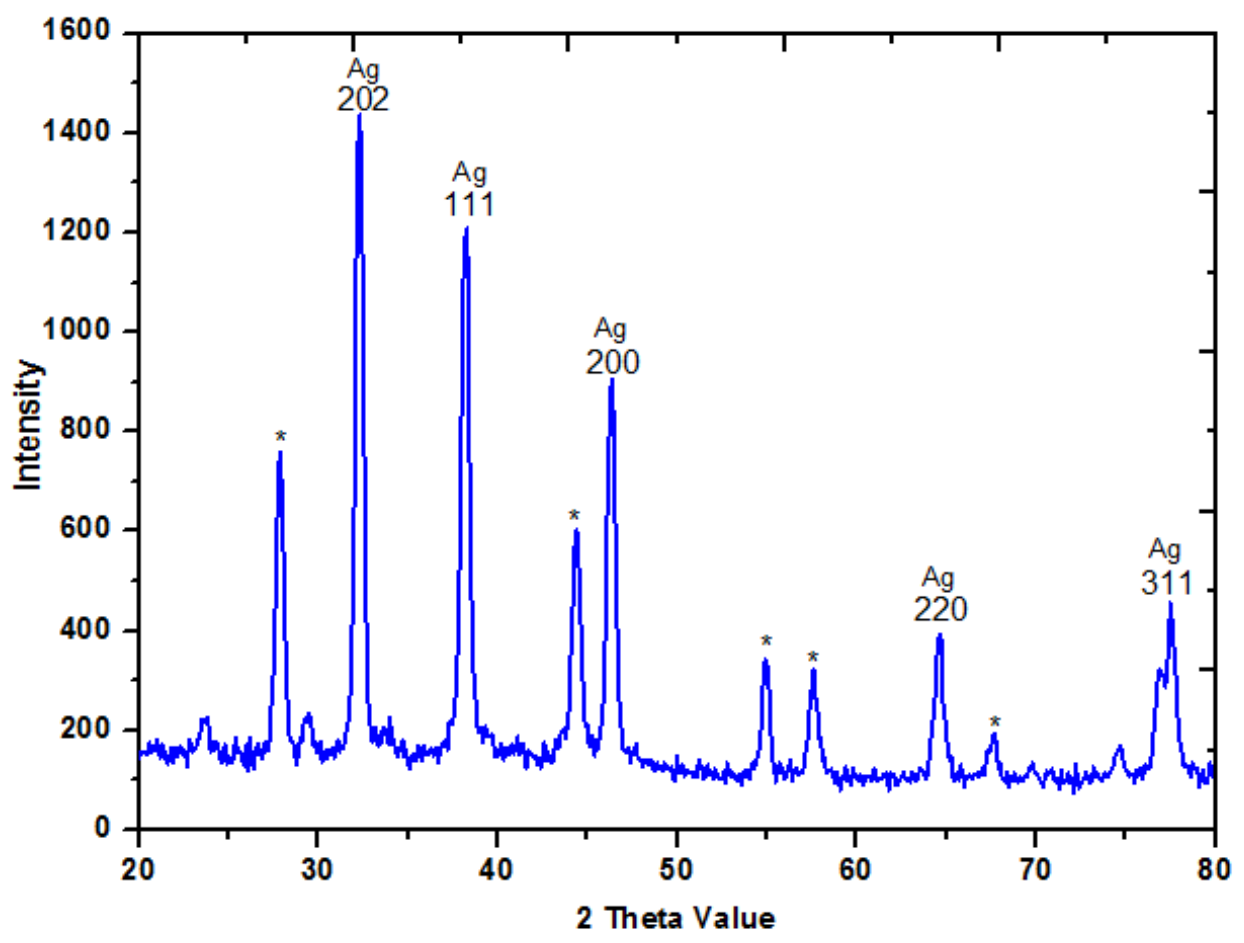


Figure 5:- SEM image of biosynthesised Silver nanoparticles

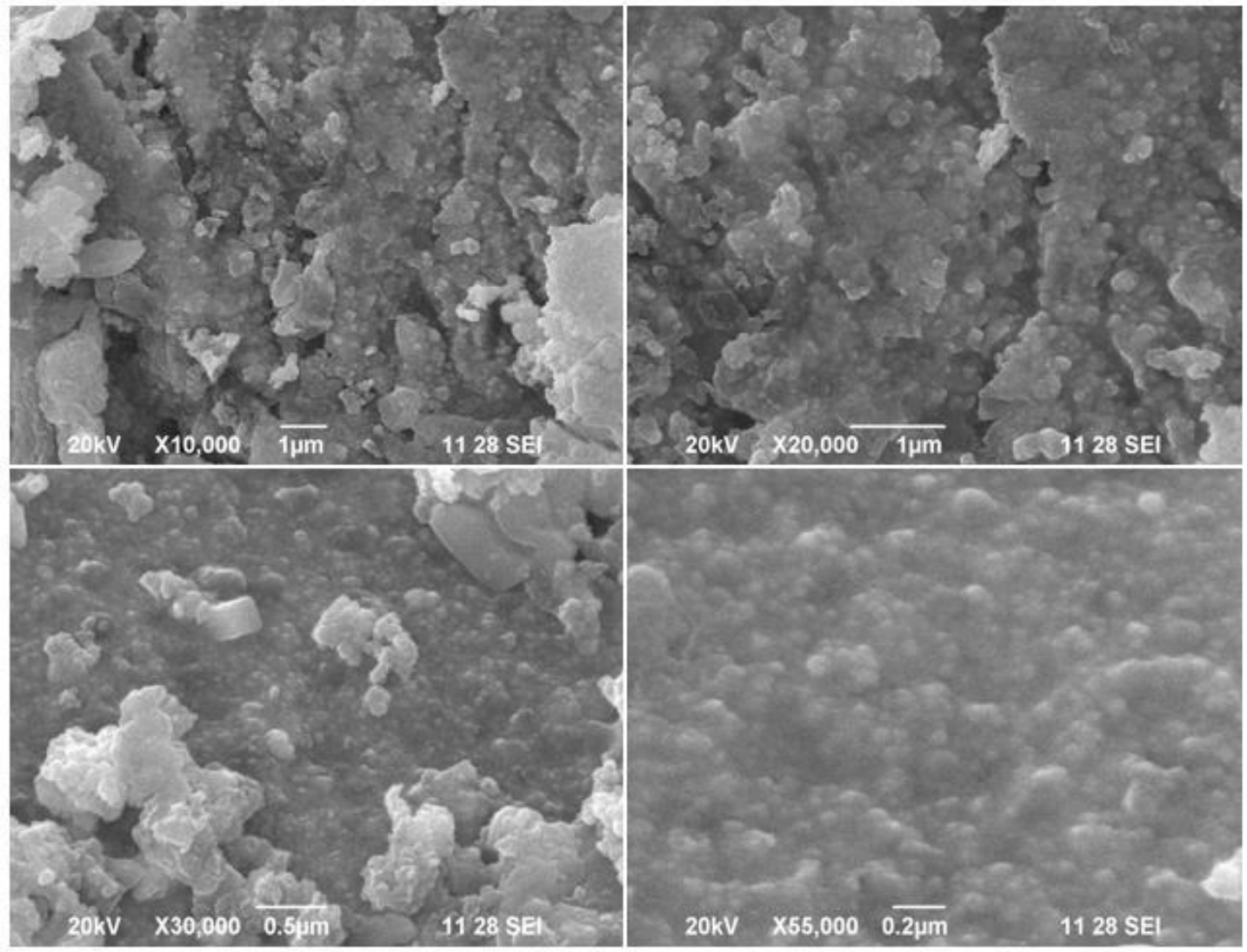

Figure 6:- EDX spectrum of Silver nanoparticles

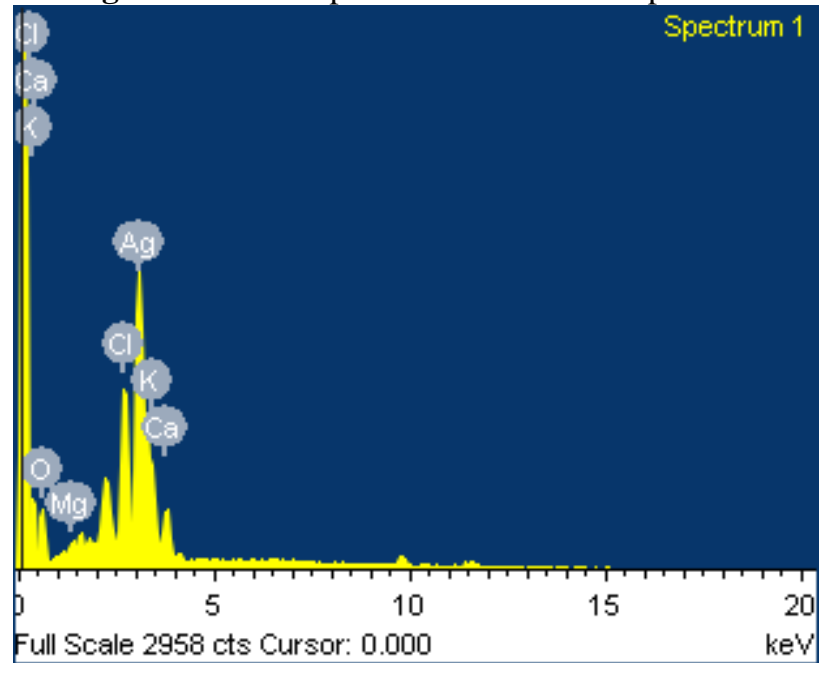


Figure 7:- Antibacterial Activity of Citrus reticulata peel mediated silver nanoparticles a) B. subtilis b) E. Coli c) K. pneumoniae d) S. paratyphi e) S. aureus and f) S. pyogenes
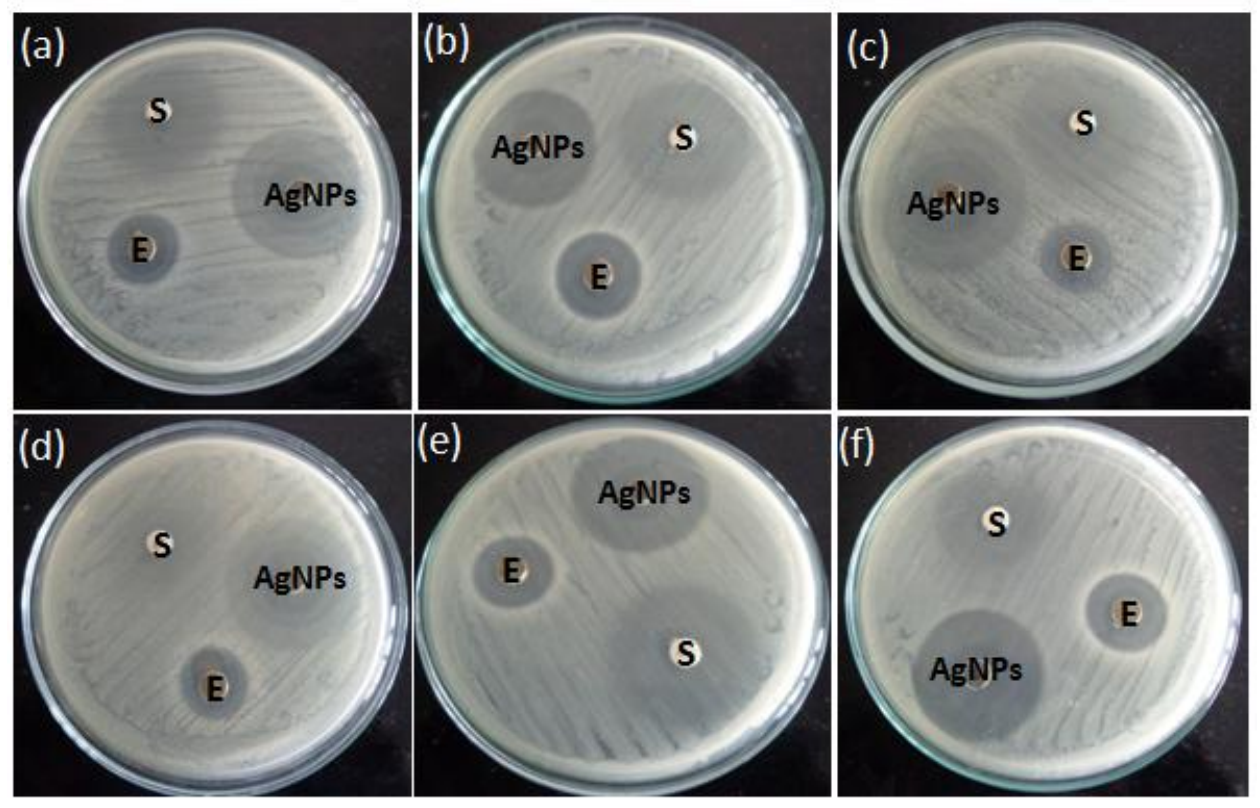

\section{Results and Discussion:-}

Eco-friendly and reliable methods have been developed to raise significance in the synthesis and application of nanoparticles that are helpful for mankind (Bhattacharya and Gupta, 2009). The major advantage of using plant extracts for silver nanoparticle synthesis is that they are easily available, safe, nontoxic in most cases and have a broad variety of metabolites that can aid in the reduction of silver ions and are quicker than microbes in the synthesis. Reduction of silver ion into silver nanoparticles for the period of exposure to the plant extracts could be followed by colour change. Silver nanoparticles exhibit yellowish-brown colour in aqueous solution due to excitation of surface plasmon vibrations (Elumalai et al., 2010). In the present study, Citrus reticulata fruit peels aqueous extract was used as reducing agent for the synthesis of $\mathrm{AgNPs}$ using $1 \mathrm{mM} \mathrm{AgNO}$. The crude extract of Citrus reticulata fruit peel was light yellow colour however after addition of $\mathrm{AgNO}_{3}$ the colour of the reaction mixture turned dark brown colour which indicated the formation of AgNPs after $6 \mathrm{~h}$ incubation period (Fig.1). This colour change indicated that the nanometric range silver ions in reaction medium have been converted to elemental silver. Metal nanoparticles such as silver have free electrons, which give rise to SPR absorption band (Veerasamy et al., 2011).

\section{UV-Vis Spectrum Analysis:-}

The green route synthesized AgNPs using Citrus reticulata fruit peel aqueous extract was confirmed by the UV-Vis spectrum analysis at different $\mathrm{nm}$ scale ranging from $300 \mathrm{~nm}$ to $800 \mathrm{~nm}$. The colour changed from light yellowish to dark brown was due to excitation of Surface Plasmon Vibration which indicated the formation of AgNPs. The Surface Plasmon broad peaks at $466 \mathrm{~nm}$ (Fig. 2) throughout the reaction clearly revealed that the green synthesized AgNPs were dispersed in the aqueous solution. It was also reported that the green synthesised silver nanoparticles using aqueous extract of Citrus reticulata peel showed the localized surface Plasmon bands at the similar regions (Elias et al., 2015).

\section{FTIR Spectrum Analysis:-}

FTIR spectrum analysis had helped to understand the nature of biomolecules present in the Citrus reticulata fruit peel extract that involved in the formation of silver nanopartilces. The FT-IR spectrum of Citrus reticulata fruit peel extract mediated green synthesized AgNPs showed sharps peak located at 3362.45, 1698.67 and light peak located at 2870.34, 2634.80, and $2361.56 \mathrm{~cm}-1$. The sharp peak at 3362.45 may to assigned to the $\mathrm{O}-\mathrm{H}$ stretch and 1698.67 may be assigned to the $\mathrm{C}-\mathrm{N}$ stretch bonding function of secondary alcohol groups. Whereas light peak at 2870.34 , 2634.80, and $2361.56 \mathrm{~cm}-1$ may be assigned to the $\mathrm{C}-\mathrm{O}, \mathrm{N}-\mathrm{H}$ and $\mathrm{O}-\mathrm{H}$ stretching that indicated the presence of phenol, amine and carboxylic acid functional group (Fig.3). The FTIR spectrum revealed that the different functional groups present at different position and functional biomolecules like phenols, alcohols and carboxylic 
acid are involved in the reduction of silver ions. Hence, the existence of these functional groups is responsible for the stabilization of synthesized silver nanoparticles and also acts as reducing and capping agent (Haytham, 2015).

\section{XRD Analysis:-}

X-ray diffraction spectrum of Citrus reticulata fruit peel mediated silver nanoparticles showed the Braggs reflections in the XRD pattern at $2 \theta=32.27,38.25,46.60,64.78$ and 78.69 These Braggs reflections clearly indicated the presence of (202), (111), (200), (220) and (311) sets of lattice planes and further on the basis that they can be indexed as face-centered-cubic (FCC) structure of silver (JCPDS file nos. 04-0783). A comparison of obtained XRD spectrum with the standard, confirmed that the silver particles formed in present experiments were in the form of nanocrystals. X-ray diffraction results clearly showed that the silver nanoparticles formed by the reduction of $\mathrm{Ag}^{+}$ions by Citrus reticulata fruit peel extract are crystalline in nature (Fig.4). The average crystallite size of green route synthesized silver nanoparticles was estimated from the full width half maximum (FWHM) of (111) reflection by using Scherrer formula, $\mathrm{D}=0.9 \lambda / \beta \cos \theta$. Where $\mathrm{D}$ is the average crystallite domain size perpendicular to the reflecting planes, $\lambda$ is the $X$-ray wavelength source $(0.1541 \mathrm{~nm}), \beta$ is the full width at half maximum and $\theta$ is the diffraction angle. It was observed that the average diameter of the silver nanoparticles crystal was about $24 \mathrm{~nm}$. Some unassigned intense diffraction peaks $(*)$, might be related to the crystallization of bioorganic phases that attached on the surface of the nanoparticles. The average particle size of silver nanoparticles synthesized by the present green method can be calculated using the Debye-Scherrer equation (Nabikhan et al., 2010).

\section{SEM-EDX Analysis:-}

Scanning electron microscopy images showed the shape of the green synthesized AgNPs using Citrus reticulata fruit peels aqueous extracts (Fig. 5). The surface morphology of the AgNPs was observed at different magnification and it revealed that orange peels extract mediated AgNPs were uniformly distributed on the surface of the cell with high agglomeration. The observation of larger silver nanoparticles may be due to the high aggregation of the smaller ones (Ranjithkumar et al., 2013). This may be due to availability of different quantity and nature of bioorganic compounds present in the aqueous extract. The SEM equipped with Energy Dispersive X-ray Spectroscopy (EDX) analysis indicated the presence of silver which was confirmed from the Ag peaks (Fig. 6). The strong signals in the silver region around $3.28 \mathrm{keV}$ confirmed the formation of AgNPs. Throughout the scanning range, some additional peaks belonging to other organic compounds present in the extract of Citrus reticulata fruit peel were also recorded and it was suggested that they were mixed precipitates of fruit peel extracts (Usha and Gladys, 2014).

\section{Antibacterial Activity:-}

Silver have long been known to have strong inhibitory and bactericidal effects, as well as a broad spectrum of antimicrobial activities which have been used for centuries to prevent and treat various diseases most notably infections. Silver nanoparticles are reported to possess anti-bacterial and anti-fungal properties (Caroling et al., 2013). In this study, antibacterial activity of Citrus reticulata fruit peels extract mediated synthesized biogenic silver nanoparticles was evaluated by using standard Zone of Inhibition (ZOI) microbiology assay against Streptococcus pyogenes, Staphylococcus aureus, Bacillus subtilis Escherichia coli, Salmonella paratyphi and Klebsiella pneumoniae and found that Citrus reticulata fruit peels extract mediated AgNPs have significant antibacterial activity against tested pathogens (Fig. 7). The maximum ZOI of Citrus reticulata fruit peel extract mediated AgNPs loaded disc was found to be $13 \mathrm{~mm}$ against Klebsiella pneumonia and Bacillus subtilis, whereas other bacterial strains like Streptococcus pyogenes, Escherichia coli, Staphylococcus aureus and Salmonella paratyphi showed 11 $\mathrm{mm}$ and $10 \mathrm{~mm}$ zone of inhibition respectively. Previous study for the silver nanoparticles synthesized using orange peel extract showed the maximum of $6 \mathrm{~mm}$ zone of inhibition against tested E. coli, Pseudomonas sp. and Salmonella sp. (Awad et al., 2014). The analysis of antibacterial activity of silver nanoparticles synthesized from Citrus sinensis showed utmost zone against E.coli $(17 \mathrm{~mm})$ at $2 \mathrm{mM} \mathrm{AgNO}_{3}$ and S.aureus $(16.5 \mathrm{~mm})$ at $2 \mathrm{mM}$ $\mathrm{AgNO}_{3}$ (Chandrasekar et al., 2015). Likewise, our present results suggested that the plant mediated metallic silver nanoparticles have been a good antibacterial activity which can be used as effective material for biological applications.

\section{Conclusion:-}

Green chemistry nanoparticles are gaining importance due to the free of toxic chemicals and provide effective synthesis of expected products in an economic manner. In this present work, we developed an environmental friendly and convenient green chemistry method for the synthesis of silver nanoparticles from fruit waste as reducing agent and Citrus reticulata fruit peels aqueous extract is found to be suitable for the production of AgNPs at room temperature by green approach. Production of AgNPs after incubation was identified by the colour change 
that occurs due to Surface Plasmon Resonance during the reaction with the organic compounds present in the Citrus reticulata fruit peels aqueous extract. The formation of AgNPs was confirmed by UV-Vis spectrum and Surface Plasmon broad peak observed nearby $466 \mathrm{~nm}$. The FTIR spectrum indicated the different functional biomolecules present at different position such as phenols, alcohols and carboxylic acid which are involved in the reduction of silver ions. XRD and SEM-EDX indicated that the Citrus reticulata fruit peels aqueous extract mediated AgNPs were uniformly distributed on the surface of the cell with high agglomeration. In addition, Citrus reticulata fruit peels aqueous extract mediated green synthesized silver nanoparticles showed excellent antibacterial properties against human pathogens at low concentration level.

\section{Acknowledgment:-}

We acknowledge our management of Dr. N. G.P. Arts and Science College and Department of Biotechnology, for providing facilities and encouragement.

\section{Reference:-}

1. Awad, M. A., Hendi, A. A., Ortashi, K. M. O., Elradi, D. F. A., Eisa, N. E., Al-lahieb, L. A., Al-Otiby, S. M., Merghani, N. M. and Awad, A. A. G. (2014): Silver nanoparticles biogenic synthesized using an orange peel extract and their use as an anti-bacterial agent. Int J Phys Sci., 9(3): 34-40.

2. Bhattacharya, D. and Gupta, R. K. (2009): Nanotechnology and potential of microorganisms. Crit Rev Biotechnol., 25: 199-204.

3. Caroling, G., Tiwari, S.K., Ranjitham, A.M. and Suja R. (2013): Biosynthesis of silver nanoparticles using aqueous Broccoli extract- Characterization and study of antimicrobial, cytotoxic effects. Asian J Pharm Clin Res., 6(4): 165-172.

4. Chandrasekar, S., Bharani devi, T., Nandhini, B. and Jayashree, S. (2015): Antibacterial activity of silver nanoparticles synthesized from Lactobacillus sp., and fruit peel extracts. World J Pharm \& Pharmaceut Sci., 4(10): 1535-1545.

5. David, E., Elumalai, E.K., Prasad, T.N.V.K.V., Venkata, K. and Nagajyothi, P.C. (2010): Green synthesis of silver nanoparticles using Euphorbia hirta L and their antifungal activities. Archives appl Sci Res., 2(6): 76-81.

6. Elias, B. S., Natiara, V. M., Fernando, A. S. and Italo, O. M. (2015): Silver nanoparticlels: green synthesis, self assembled nanostructures and their application as SERS substrates. New J Chem., 39: 2839-2846.

7. Elumalai, E.K., Prasad, T.N.V.K.V., Hemachandran, J., Therasa, S.V., Thirumalai, T. and David, K. (2010): Extracellular synthesis of silver nanoparticles using leaves of Euphorbia hirta and their antibacterial activities. J Pharm Sci and Res., 2: 549-554.

8. Gurunathan, S., Kalishwaralal, K., Vaidyanathan, R., Deepak, V., Pandian, S.R.K. and Muniyandi J. (2009): Biosynthesis, purification and characterization of silver nanoparticles using Escherichia coli. Colloids surf B., 74: 328-335.

9. Haverkamp, R.G. and Marshall, A.T. (2009): The mechanism of metal nanoparticles formation in plants: limits on accumulation. J Nanopart Res., 11(6): 1453-1463.

10. Haytham M. M. I. (2015): Green synthesis and characterization of silver nanoparticles using banana peel extract and their antimicrobial activity against representative microorganisms. J Radiat Res and Appl Sci., 8(3): 265275.

11. Jain, D., Daima, H.K., Kachhwaha, S. and Kothari, S.L. (2009): Synthesis of plant-mediated silver nanoparticles using papaya fruit extract and evaluation of their anti-microbial acrivities. Dig J Nanomater Biostruct., 4: 723-727

12. Karnani, R.L. and Abhay Chowdhary. (2013): Biosynthesis of Silver nanoparticles by eco- friendly method. Indian J Nanosci., ; 1(2):25-31.

13. Madhumitha, G. and Selvaraj, M. R. (2013). Devastated Crops: Multifunctional efficacy for the production of Nanoparticles. J nanopart., 1-12

14. Mukunthan, K. and Balaji, S. (2012): Cashew apple juice (Anacardium occidentale L.) speeds up the synthesis of silver nanoparticles. Int J Green Nanotech., 4: 71-79.

15. Nabikhan, A., Kandasamy, K., Raj, A. and Alikunhi, N. M. (2010): Synthesis of antimicrobial silver nanoparticles by callus and leaf extracts from saltmarsh plant Sesuvium portulacastrum L. Colloids Surf B., 79: 488-493.

16. Parashar, V., Parashar, R., Sharma, B. and Pandey, A.C. (2009): Parthenium leaf extract mediated synthesis of silver nanoparticles: a novel approach towards weed utilization. Dig J Nanomater Biostruct., 4: 45-50. 
17. Ranjithkumar, R., Selvam, K. and Shanmugavadivu, M. (2013): Green Synthesis of Silver Nanoparticles Using Areca Nut Extract for Enhanced Antibacterial Activity. J Green Sci Technol., 1: 102-106.

18. Sathyavathi, R., Balamurali Krishna, M., Venugopal Rao, S., Saritha, R. and Narayana Rao, D. (2010): Biosynthesis of Silver Nanoparticles using Coriandrum sativum leaf extract and their Application in Nonlinear Optics. Adv Sci Lett., 3(2): 1-6.

19. Singh, M., Singh, S., Prasad, S. and Gambhir, I.S. (2008): Nanotechnology in medicine and antibacterial effect of silver nanoparticles. Dig J Nanomater Biostruct., 3: 115-122.

20. Usha, C. and Gladys D.A.R. (2014): Biogenic synthesis of silver nanoparticles by Acacia nilotica and their antibacterial activity. Int J Scientific Res., 3(6): 27-29.

21. Veerasamy, R., Xin, T. Z., Gunasagaran, S., Xiang, T. F. W., Yang, E. F. C., Jeyakumar N. and Dhanaraj, S. A. (2011): Biosynthesis of silver nanoparticles using mangosteen leaf extract and evaluation of their antimicrobial activities. J Saudi Chem Soc., 15: 112-120. 